NASA Technical Memorandum 109125

22323

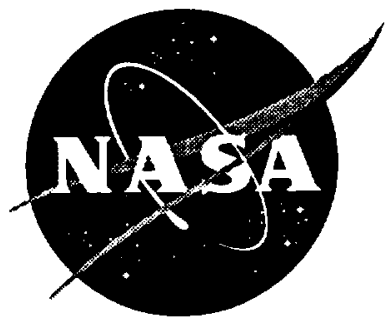

\title{
Cross-Surface Interface Element For Coupling Built-up Structural Subdomains
}

C. G. Dávila

NRC Resident Research Associate

Langley Research Center, Hampton, Virginia

J. B. Ransom

Langley Research Center, Hampton, Virginia

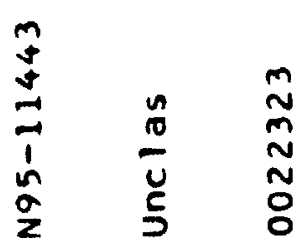

M. A. Aminpour

Old Dominion University, Norfolk, Virginia

July 1994

National Aeronautics and

Space Administration

Langley Research Center

Hampton, Virginia 23681-0001

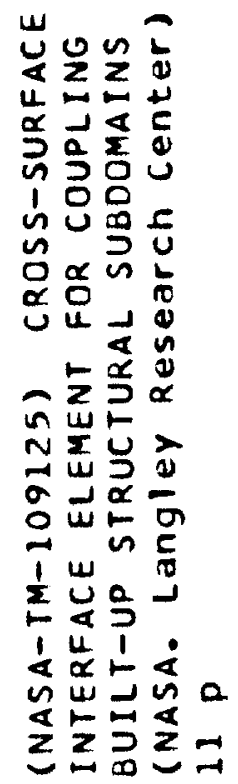




\section{CROSS-SURFACE INTERFACE ELEMENT FOR COUPLING BUILT-UP STRUCTURAL SUBDOMAINS}

\author{
Carlos G. Dávila* \\ National Research Council Associate \\ at NASA Langley Research Center \\ Hampton, VA 23681
}

\author{
Jonathan B. Ransom** \\ NASA Langley Research Center \\ Hampton, VA 23681
}

\author{
Mohammad A. Aminpour ${ }^{* * *}$ \\ Old Dominion University \\ Department of Aerospace Engineering \\ Norfolk, VA 23508
}

\begin{abstract}
A new finite element for coupling built-up shell substructures is presented. The present work extends the hybrid variational formulation of the interface element developed by Aminpour and Ransom to permit coupling between two intersecting substructures. Designed for the assembly of independent built-up finite element models, this technique provides a level of modeling flexibility previously unavailable.
\end{abstract}

\section{Introduction}

The analysis of modern aerospace and ground vehicle structures relies heavily on the finite element method. While detailed and accurate stress predictions can be made for even the most geometrically complex structural assemblies, practical limitations in mesh size and mesh discretization often prevent the method's use in the design phase. Indeed, the finite element method can overwhelm available resources in two major ways. Not only can the size of a practical model quickly reach the limits of the accessible computational hardware, but the generation and validation of the mesh are likely to require a more than considerable effort. In practice, many useful analysis and design studies that could lead to lower-cost designs are precluded.

One example where meshing can become extraordinarily costly is structural analysis in multi-disciplinary. optimization. Shape optimization in aircraft design involves iterations on aerodynamic and structural

\footnotetext{
* NRC Resident Research Associate.

** Aerospace Engineer.

*** Associate Professor.
}

analyses. These optimizations are carried out concurrently, with both analyses sharing a common description of the airframe. This parameterized three-dimensional description is used to develop the aero grid and the structural finite element mesh of the skin. Although similar to each other, these two meshes are so fundamentally different in purpose that the same discretization is not used for aerodynamic and structural analyses. Indeed, aero grids are only discretizations of the boundary of the aircraft's volume, and their size and density is often changed during an analysis. The structural mesh of the skin, on the other hand, is tightly constrained by the locations of its internal components. Node-to-node compatibility between structural components such as bulkheads, ribs, stiffeners and the aircraft skin dictate constraints on the finite element mesh that do not have a counterpart in the aero grid. Consequently, structural skin meshes are, at best, difficult and expensive to generate.

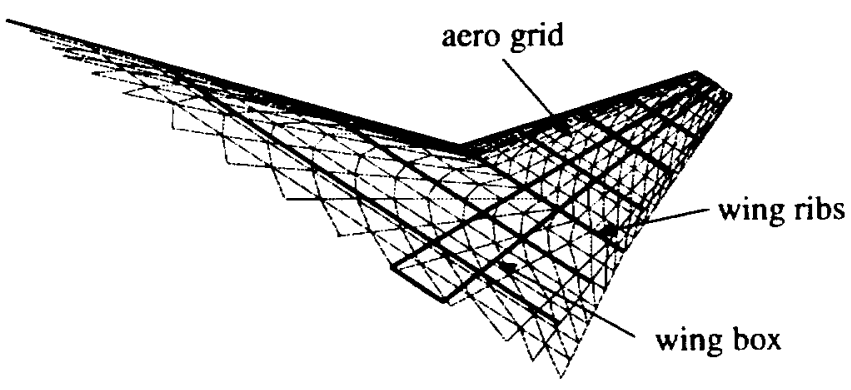

Figure 1. The generation of structural models would be streamlined if the wing box and ribs could be connected to the aero grid.

On the other hand, if the internal structural components of a wing could be coupled to the elements of the skin along arbitrary intersection curves, as illustrated in 
Fig. 1, the constraints that make structural meshes difficult to generate would be eliminated. Furthermore, the aero grid may now be suitable as a structural mesh for the skin. This would eliminate the need for remeshing, and provide the added advantage of relieving the translation losses that result from interpolating the aerodynamic loads from the aero grid to the structural mesh.

Detailed finite element models are not efficient in the design phase because they are computationally intensive and require extensive modeling effort. The interface element developed by Aminpour et al. ${ }^{1}$ and Ransom et al. ${ }^{2}$ addresses some of the difficulties associated with mesh generation and optimization. This interface element was developed to enforce compatibility on the boundaries of independently modeled shell substructures so that the need for complex mesh transitioning is eliminated. Because it allows the finite element domain to be subdivided into various substructures with different element discretizations, the interface element provides modeling flexibility and allows the generation of independently optimal meshes.

Unlike other coupling methods based on mortar elements ${ }^{3}$ or spline fit ${ }^{4}$, the interface element based on a hybrid variational formulation has been shown to be robust and accurate. It preserves solution accuracy of displacements and stresses across the common substructure boundaries.

However, the interface element of Refs. 1 and 2 lacks generality in one essential respect. It is designed for coupling shell substructures sharing a common edge on which all the connected finite element nodes of the connected substructures lie (Figs. $2 \mathrm{a}$ and $2 \mathrm{~b}$ ). The present formulation extends this concept to include the more general problem of coupling across element faces, as shown in Fig 3 . In this figure, substructure $\Omega_{1}$ is coupled across the surface of substructure $\Omega_{2}$. For simplicity, we refer hereafter to $\Omega_{1}$ as the stiffener, and to $\Omega_{2}$ as the skin.

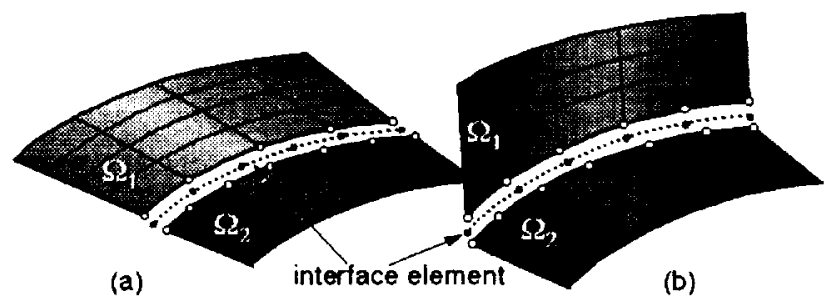

Figure 2. The interface element of Refs. 1 and 2 allows coupling of independently modeled substructures along a common edge.

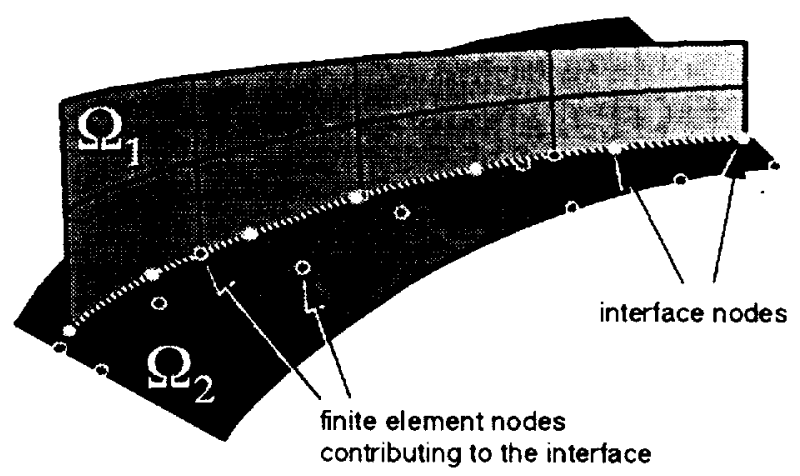

Figure 3. A new cross-surface interface element is used to couple substructure $\Omega_{1}$ across the surface of substructure $\Omega_{2}$.

The objective of this work is to develop an interface element with cross-surface capabilities, and to evaluate the accuracy and usefulness of this element. Therefore, it was considered of vital importance that even the prototype for this element possess a fully automated capability for determining the path of the stiffener on the surface of the skin. This capability includes the determination of the elements and degrees of freedom that contribute to the interface. The first two examples presented in this paper illustrate the accuracy of the method under a "worst-case scenario", as well as under more normal conditions. The last problem is an example of a parametric study in which the location of structural components is varied without modifying the mesh.

\section{Cross-Surface Interface Element}

The hybrid variational formulation used in the interface element was presented in detail by Aminpour et al. ${ }^{1}$ and is briefly described here. The formulation employs an integral form for the compatibility between the interface and the subdomains. For two subdomains $\Omega_{1}$ and $\Omega_{2}$ sharing a common interface, $s$, the modified total potential energy is written as

$$
\Pi=\Pi_{\Omega_{1}}+\Pi_{\Omega_{2}}+\int_{s} \lambda_{1}^{T}\left(v-u_{1}\right)+\lambda_{2}^{T}\left(v-u_{2}\right) d s
$$

where $\lambda_{1}$ and $\lambda_{2}$ are vectors of the Lagrange multipliers corresponding to subdomains $\Omega_{1}$ and $\Omega_{2}$, respectively. The displacement vector of the interface element, $\boldsymbol{v}$, is allowed to be independent of the displacement vectors $u_{1}$ and $u_{2}$, of the subdomains. It can be shown that the stationary condition for the total potential energy results in the Euler equations

$$
\delta \Pi=0 \Rightarrow \begin{cases}\lambda_{i}=(\sigma n)_{i} & , \quad i=1,2 \\ \lambda_{1}+\lambda_{2}=0 & \\ u_{i}=v \quad, i=1,2 & \text { on } s\end{cases}
$$


where $n$ represents the outward unit normal to the subdomains along their interfaces, $\sigma$ is the stress tensor, and the Lagrange multipliers $\lambda_{1}$ and $\lambda_{2}$ represent the tractions at the interfaces. Equation 2 is an expression of displacement continuity and traction reciprocity, which guarantees that equilibrium is maintained across the interface.

The interpolations for the finite element displacements, interface displacements, and interface tractions are, respectively,

$$
\left.\begin{array}{l}
\boldsymbol{u}_{i}=N_{i} \hat{\boldsymbol{q}}_{i} \\
\boldsymbol{v}=T \hat{\boldsymbol{q}}_{s} \\
\lambda_{i}=R_{i} \hat{\lambda}_{i}
\end{array}\right\} \quad i=1,2 \text { for } \Omega_{j} \text { and } \Omega_{2}
$$

where $\hat{\boldsymbol{q}}_{i}$, and $\hat{\boldsymbol{q}}_{s}$ are the nodal degrees of freedom corresponding to $\boldsymbol{u}_{i}$ and $\boldsymbol{v}$, respectively, $\lambda_{i}$ are the unknown coefficients for the Lagrange multipliers, $\lambda_{i}, N_{i}$ is the matrix of the finite element shape functions on substructure $\Omega_{i}, T$ is the matrix of shape functions for the (independent) interface displacements, and $R_{i}$ is the matrix of interpolating functions for the Lagrange multipliers on substructure $\Omega_{i}$.

Taking the first variation of the modified total potential energy, Eq. 1, with respect to the independent degrees of freedom, and setting the variation to zero yields the system of equations

$$
\left[\begin{array}{ccccc}
K_{1} & 0 & 0 & M_{1} & 0 \\
0 & K_{2} & 0 & 0 & M_{2} \\
0 & 0 & 0 & G_{1} & G_{2} \\
M_{1}^{T} & 0 & G_{1}^{T} & 0 & 0 \\
0 & M_{2}^{T} & G_{2}^{T} & 0 & 0
\end{array}\right]\left\{\begin{array}{l}
\hat{\boldsymbol{q}}_{1} \\
\hat{\boldsymbol{q}}_{2} \\
\hat{\boldsymbol{q}}_{s} \\
\hat{\lambda}_{1} \\
\hat{\lambda}_{2}
\end{array}\right\}=\left\{\begin{array}{c}
\hat{f}_{1} \\
\hat{\boldsymbol{f}}_{2} \\
0 \\
0 \\
0
\end{array}\right\}
$$

where $K_{1}$ and $K_{2}$ are the assembled stiffness matrices of the uncoupled subdomains, $\hat{f}_{i}$ are the nodal force vectors, and the coupling submatrices $M_{i}$ and $G_{i}$ are composed of integrals of the interpolation matrices $N_{i}, T$, and $R_{i}$ along the interface, $s$. They are defined as

$$
M_{i}=-\int_{s} N_{i}^{T} R_{i} d s \quad \text { and } \quad G_{i}=\int_{s} T^{T} R_{i} d s
$$

For simplicity, the following development is for a single degree-of-freedom. The same pattern is repeated for the remaining degrees of freedom. Furthermore, the following computation of the integrals in Eqs. 5 is shown for one four-node skin element along the path of the stiffener. The full matrices are obtained by adding the terms for all the elements crossed by the stiffener. The computation of the coupling terms for elements other than four-node is not attempted here.
For a four-node element, $N_{i}=\left\{n_{1}, n_{2}, n_{3}, n_{4}\right\}_{i}$, where the bilinear interpolation functions are

$$
\begin{aligned}
& n_{1}=\frac{1}{4}(1-\zeta)(1-\eta) \\
& n_{2}=\frac{1}{4}(1+\zeta)(1-\eta) \\
& n_{3}=\frac{1}{4}(1+\zeta)(1+\eta) \\
& n_{4}=\frac{1}{4}(1-\zeta)(1+\eta)
\end{aligned}
$$

The distribution of the tractions, $\lambda_{i}$, is assumed to be constant over the face of a four-node element, so the corresponding interpolation function matrix is $R_{i}=1$. Therefore, the form of the matrix $M_{i}$ is $M_{i}=\left\{m_{1}, m_{2}, m_{3}, m_{4}\right\}_{i}^{T}$, where $m_{l}$ is a function of $n_{l}$.

For the original interface element, illustrated in Fig. $4 \mathrm{a}$, the integration of matrix $M_{i}$ in Eq. 5 is only in $\eta$, from $\eta=-1$ to $\eta=+1$. Substituting $\zeta=-1$ into Eqs. 6, and noting that $d s=\frac{s_{2}-s_{1}}{2} d \eta$, the terms in the coupling matrix $M_{i}$ are

$$
\begin{aligned}
& m_{1}=-\frac{1}{2} \int_{s_{1}}^{s_{2}}(1-\eta) \cdot 1 d s=-\frac{\left(s_{2}-s_{1}\right)}{2} \\
& m_{2}=-\frac{1}{4} \int_{s_{1}}^{s_{2}} 0 \cdot(1-\eta) \cdot 1 d s=0 \\
& m_{3}=-\frac{1}{4} \int_{s_{1}}^{s_{2}} 0 \cdot(1+\eta) \cdot 1 d s=0 \\
& m_{4}=-\frac{1}{2} \int_{s_{1}}^{s_{2}}(1+\eta) \cdot 1 d s=-\frac{\left(s_{2}-s_{1}\right)}{2}
\end{aligned}
$$

which confirms that the degrees of freedom at nodes 2 and 3 (corresponding to $m_{2}$ and $m_{3}$ ) do not contribute to the interface.

In the general case shown in Fig. $4 \mathrm{~b}$, however, the interface crosses the finite element's face, and all nodes in the element contribute to the interface. The interpolation is expressed in terms of both natural coordinates, $\eta$ and $\zeta$. The first term of $M_{i}$ is

$$
m_{1}=-\frac{1}{4} \int_{s_{1}}^{s_{2}}(1-\zeta)(1-\eta) \cdot 1 d s
$$

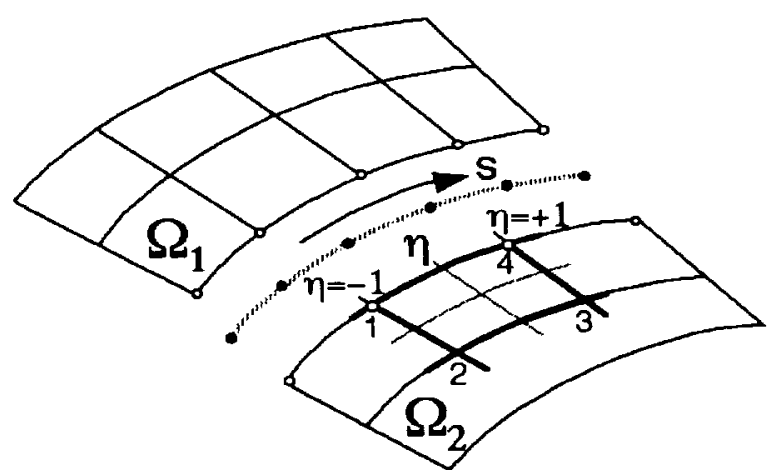

Figure 4a. Integration path along the edge of an element. 


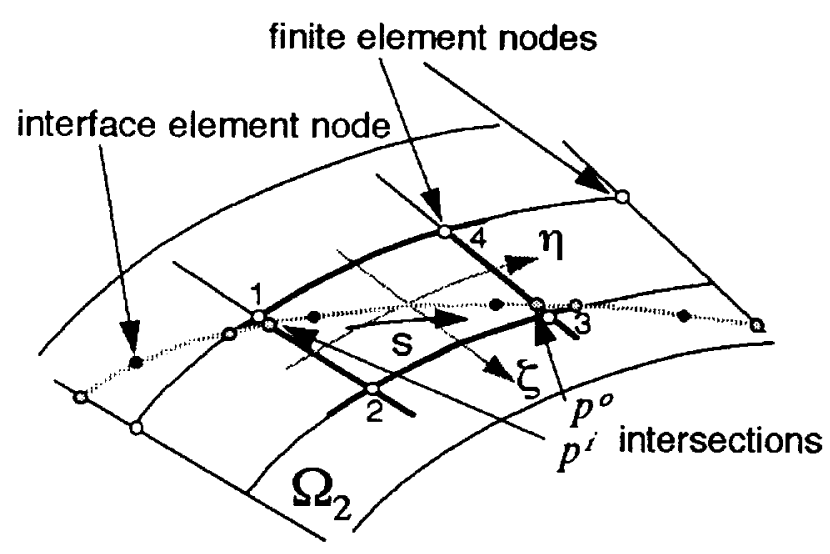

Figure 4b. Integration path across the surface of an element.

To perform the integration in Eq. 8 it is necessary to relate the element's natural coordinates, $\zeta$ and $\eta$, and the path increment, $d s$, to a single variable. This requires a complete description of the intersection path that is generated by the stiffener on the elements in the skin. One simplifying assumption that allows closedform solution of this integration is illustrated in Fig. 5. It is assumed that the interface is contained in a plane $\Psi$, which is referred to as the plane of the interface. The equation of this plane is expressed in terms of its normal, $\boldsymbol{n}=\left\{n_{x}, n_{y}, n_{z}\right\}$ and offset, $d$

$$
n_{x} x+n_{y} y+n_{z} z=d
$$

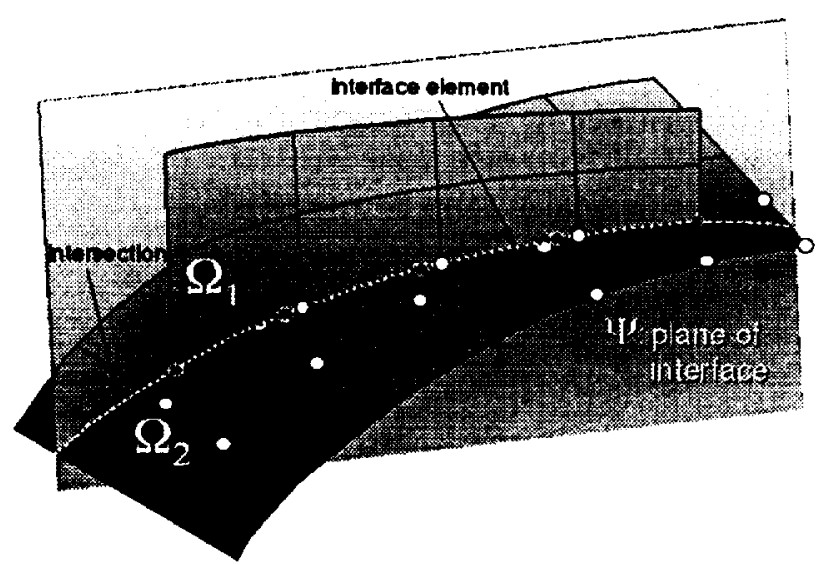

Figure 5. A closed-form solution is obtained by assuming that the interface is contained in a plane which intersects the skin (substructure $\Omega_{2}$ ).

The first step in the automated definition phase of the interface element consists of computing the four coefficients in Eq. 9 from the nodes on the stiffener (provided by the user) which define the location of the interface. The interface plane is used to locate the elements in the skin that lie along the path of the stiffener. For these elements, two and only two of the following four intersection conditions are valid:

$$
\begin{array}{ll}
\eta=-1, & -1 \leq \zeta \leq 1 \\
\zeta=+1, & -1 \leq \eta \leq 1 \\
\eta=+1, & -1 \leq \zeta \leq 1 \\
\zeta=-1, & -1 \leq \eta \leq 1
\end{array}
$$

For instance, on the edge between nodes 1 and 2 in Fig. $4 b, \eta=-1$ (first condition in Eqs. 10). Substituting this condition into the interpolation functions, Eqs. 6, and then into the equation of the intersecting plane, Eq. 9 , and solving for $\zeta$ results in

$$
\zeta=\frac{n_{x}\left(x_{1}+x_{2}\right)+n_{y}\left(y_{1}+y_{2}\right)+n_{z}\left(z_{1}+z_{2}\right)-2 d}{n_{x}\left(x_{1}-x_{2}\right)+n_{y}\left(y_{1}-y_{2}\right)+n_{z}\left(z_{1}-z_{2}\right)}
$$

If $-1 \leq \zeta \leq+1$, the first condition in Eqs. 10 is satisfied. One more condition out of the remaining three must also be satisfied for a valid intersection. After sorting the elements along the intersection of the plane $\Psi$ and the skin, it is possible to identify and eliminate the elements outside the path of the interface. All of the remaining elements contribute to the interface, and they possess a point through which the path of the interface enters the element and a point through which it exits the element. We refer to the natural coordinates of these two points as $\zeta^{i}, \eta^{i}$ and $\zeta^{\circ}, \eta^{\circ}$, respectively, and to the corresponding Cartesian coordinates as $\boldsymbol{p}^{i}=\left\{p_{x}^{i}, p_{y}^{i}, p_{z}^{i}\right\}$ and $\boldsymbol{p}^{o}=\left\{p_{x}^{o}, p_{y}^{o}, p_{z}^{o}\right\}$ (see Fig. 4b).

The relation between $\zeta$ and $\eta$ needed to perform the integration of Eq. 8 is obtained as follows. The coordinates of a point on an element can be written using the element's interpolation as:

$$
\begin{aligned}
4 x= & \zeta \eta\left(x_{1}-x_{2}+x_{3}-x_{4}\right)+\zeta\left(-x_{1}+x_{2}+x_{3}-x_{4}\right) \\
& +\eta\left(-x_{1}-x_{2}+x_{3}+x_{4}\right)+\left(x_{1}+x_{2}+x_{3}+x_{4}\right) \\
4 y= & \zeta \eta\left(y_{1}-y_{2}+y_{3}-y_{4}\right)+\zeta\left(-y_{1}+y_{2}+y_{3}-y_{4}\right) \\
& +\eta\left(-y_{1}-y_{2}+y_{3}+y_{4}\right)+\left(y_{1}+y_{2}+y_{3}+y_{4}\right) \\
4 z= & \zeta \eta\left(z_{1}-z_{2}+z_{3}-z_{4}\right)+\zeta\left(-z_{1}+z_{2}+z_{3}-z_{4}\right) \\
& +\eta\left(-z_{1}-z_{2}+z_{3}+z_{4}\right)+\left(z_{1}+z_{2}+z_{3}+z_{4}\right)
\end{aligned}
$$

where $x_{i}, y_{i}$, and $z_{i}, i=1,4$, are the coordinates of the nodes of the element. Substituting Eq. 12 into the equation of the plane of the interface, Eq. 9, gives

$$
\zeta=-\frac{\eta t_{3}+t_{4}}{\eta t_{1}+t_{2}} \quad \text { or } \quad \eta=-\frac{\zeta t_{2}+t_{4}}{\zeta t_{1}+t_{3}}
$$


where the constants $t_{i}$ are defined:

$$
\begin{aligned}
& t_{1}=n_{x}\left(x_{1}-x_{2}+x_{3}-x_{4}\right)+n_{y}\left(y_{1}-y_{2}+y_{3}-y_{4}\right) \\
&+n_{z}\left(z_{1}-z_{2}+z_{3}-z_{4}\right) \\
& t_{2}=n_{x}\left(-x_{1}+x_{2}+x_{3}-x_{4}\right)+n_{y}\left(-y_{1}+y_{2}+y_{3}-y_{4}\right) \\
&+n_{z}\left(-z_{1}+z_{2}+z_{3}-z_{4}\right) \\
& t_{3}=n_{x}\left(-x_{1}-x_{2}+x_{3}+x_{4}\right)+n_{y}\left(-y_{1}-y_{2}+y_{3}+y_{4}\right) \\
& \quad+n_{z}\left(-z_{1}-z_{2}+z_{3}+z_{4}\right) \\
& t_{4}=n_{x}\left(x_{1}+x_{2}+x_{3}+x_{4}\right)+n_{y}\left(y_{1}+y_{2}+y_{3}+y_{4}\right) \\
& \quad+n_{z}\left(z_{1}+z_{2}+z_{3}+z_{4}\right)-4 d
\end{aligned}
$$

Finally, the incremental variable $d s$ in Eq. 8 can be expressed in terms of the incremental coordinates as

$$
d s=\sqrt{d x^{2}+d y^{2}+d z^{2}}
$$

In the case of a four-node element, we can write the linear relations between the Cartesian intersection coordinates and the path increments as

$$
\frac{d x}{\left(p_{x}^{\prime \prime}-p_{x}^{i}\right)}=\frac{d y}{\left(p_{y}^{o}-p_{y}^{i}\right)}=\frac{d z}{\left(p_{z}^{o}-p_{z}^{i}\right)}
$$

from which two of the increments may be expressed in terms of a third. For instance, if $\left(p_{x}^{o}-p_{x}^{i}\right)$ in Eq. 16 is nonzero, then $\mathrm{Eq} .15$ gives

$$
d s=\sqrt{1+\alpha^{2}+\beta^{2}}|d x|
$$

where $\alpha$ and $\beta$ are the appropriate ratios of the denominators in Eqs. 16. The term $d x$ is obtained from the first term in Eqs. 12. Therefore, we can write

$$
\begin{aligned}
& d s=\frac{\sqrt{1+\alpha^{2}+\beta^{2}}}{4} \mid\left(\left(x_{1}-x_{2}\right) \frac{(1-\eta)\left(t_{2} t_{3}-t_{1} t_{4}\right)}{\left(\eta t_{1}+t_{2}\right)^{2}}\right. \\
&-\left(x_{3}-x_{4}\right) \frac{(1+\eta)\left(t_{2} t_{3}-t_{1} t_{4}\right)}{\left(\eta t_{1}+t_{2}\right)^{2}} \\
&-\left(x_{1}-x_{4}\right)\left(1+\frac{\eta t_{3}+t_{4}}{\eta t_{1}+t_{2}}\right) \\
&\left.-\left(x_{2}-x_{3}\right)\left(1-\frac{\eta t_{3}+t_{4}}{\eta t_{1}+t_{2}}\right)\right) d \eta \mid
\end{aligned}
$$

Using (18), and (13), it can be shown that the closed form solution for the integral in Eq. 8 is

$$
\begin{aligned}
& m_{1}=A\left(\eta_{0}-\eta_{i}\right)+B\left(\eta_{0}^{2}-\eta_{i}^{2}\right) \\
& +C\left(\frac{1}{\left(t_{2}+t_{1} \eta_{i}\right)^{2}}-\frac{1}{\left(t_{2}+t_{1} \eta_{o}\right)^{2}}\right) \\
& +D\left(\frac{1}{\left(t_{2}+t_{1} \eta_{i}\right)}-\frac{1}{\left(t_{2}+t_{1} \eta_{o}\right)}\right)+E \log \left(\frac{t_{2}+t_{1} \eta_{i}}{t_{2}+t_{1} \eta_{o}}\right)
\end{aligned}
$$

where $A, B, C, D$ and $E$ are constants which depend only on the element's nodal coordinates. Similar results are obtained for the remaining three terms, $m_{2}, m_{3}$, and $m_{4}$.

\section{Results and Discussion}

The following examples were developed to evaluate the accuracy and limitations of the cross-surface interface element. A benchmark problem is presented first to assess the performance of the elements under a "worst case" condition, i.e., when the interface produces a severe discontinuity in the shell elements under the path of the interface. The second example illustrates the simultaneous use of four interface elements to couple blade stiffeners to a composite flat plate. Finally, the last problem consists of a framed fuselage panel with two curved interfaces. The use of interface elements in parametric studies is illustrated with two solutions corresponding to different placements of the frames.

\section{Case 1: Cantilevered plate with stiffener support}

The most demanding problems for cross-surface coupling involve a severe discontinuity in the transverse forces of the shell elements in the skin. The following "worst-case scenario" was designed to introduce a discontinuity which the shell elements in the skin cannot accomodate.

This benchmark problem consists of a cantilevered narrow plate. The plate is supported at $3 / 4$ of its length by a transverse stiffener which is clamped at its free end, as shown in Fig. 6. A load of $1.0 \mathrm{lb} /$ in is applied at one end of the plate. Young's modulus is $E=10^{6} \mathrm{psi}$, the thickness is 0.1 in., and Poisson's ratio is taken to be $v=0$ to achieve pure cylindrical bending.

The mesh of the stiffener consists of three fournode elements along the width and two along the height. When the number of elements along the length of the plate is not a multiple of four, the elements under the stiffener are in the region of the model where the bending moment changes from its maximum value on the left of the stiffener, to zero, on the right of the stiffener. Since the four-node elements used here can only represent a constant bending moment $M_{x}$ and transverse shear force $Q_{x}$ along the $x$ axis, approximation errors result, as shown in Fig. 6. This case represents the most stringent interface problem, since the stiffener introduces a severe discontinuity in the moment and transverse force for the elements on the plate. 

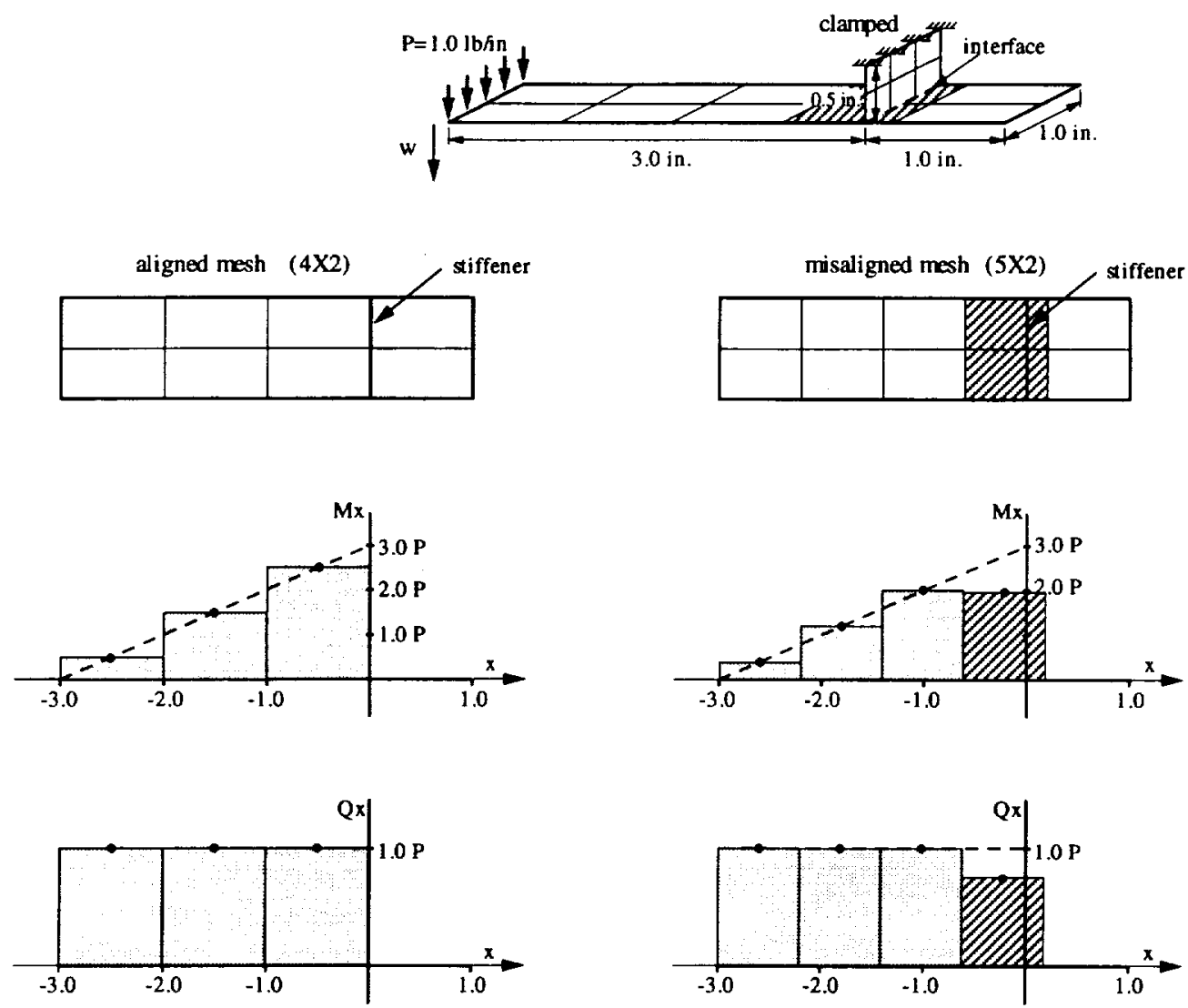

Figure 6. Cantilevered plate with transverse stiffener under tip load.

The plate's tip deflection is shown in Fig. 7 versus the number of elements along the span of the plate. When the number of elements is a multiple of four, the stiffener intersects along the edges of elements in the plate, and the solution is equivalent to the reference solution (i.e., model with no interface element). However, the convergence is not monotonic, and when the stiffener intersects near the center of an element on the plate, as for $\mathrm{N}=6$, the error reaches a maximum (12\%). It can also be observed that the solution is always bracketed between the two closest standard finite element models (i.e., the models with no interface element). The lower bracket consists of a model where the stiffener is relocated to the left edge of the intersected shell elements, and the upper bracket is one where the stiffener is placed at the right edge of the intersected shell element. As expected, the bracket narrows as the mesh in the plate is refined. It can be observed that the error in the coupled model is always much smaller than the bracket. In other words, the error introduced by the interface element is much smaller than the error that would result from attaching the stiffener to the closest element edge.

It is important to note that the oscillation in the error is not caused by the interface element, but rather, by the finite element discretization of the plate. A similar error would occur, for instance, if one were to place a line load across the shell elements in a standard finite element mesh with no interface.

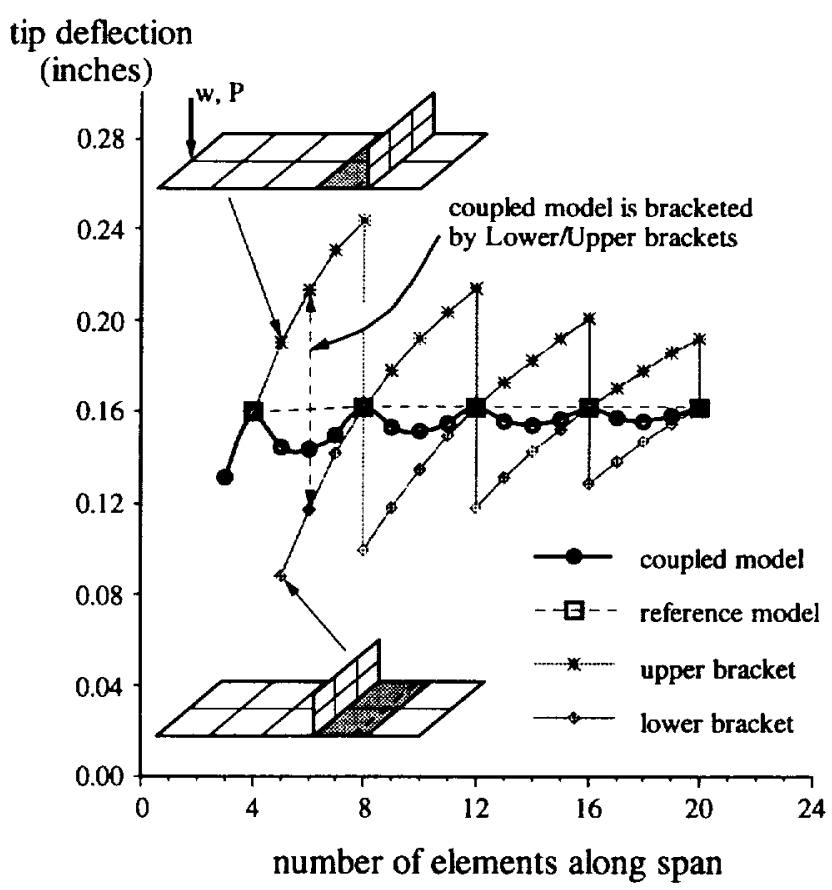

Figure 7. Tip displacements for cantilevered plate with stiffener support. The convergence of the solution is not monotonic. 


\section{Case 2: Blade-stiffened composite panel with a hole}

A linear stress analysis of a flat, blade-stiffened graphite-epoxy panel loaded in axial compression is performed. The panel is $30 \mathrm{in.} \mathrm{long,} \mathrm{the} \mathrm{width} \mathrm{is} 11.5 \mathrm{in}$., the stiffener spacing is 4.5 in., the stiffener height is 1.4 in., and the cutout radius is 1 in. All three bladestiffeners have identical cross-sections. The loading is uniform axial compression in the $x$ direction. All degrees of freedom of the loaded ends of the panel are constrained except for the motion in the axial direction. The material system used for the panel is T300/5208 graphite-epoxy unidirectional tapes with a nominal ply thickness of 0.0055 in.

The panel skin is a 25-ply symmetric laminate $\left(\left[ \pm 45 / 0_{2} / \mp 45 / 0_{3} / \pm 45 / 0 / \overline{0}\right]\right)_{\mathrm{s}}$ and the blade stiffeners are 24-ply symmetric laminates $\left(\left[ \pm 45 / 0_{10}\right]\right)_{s}$.

Since no theoretical solution exists for this configuration, a reference solution is obtained by using a finite element model which has no interface element. This reference finite element model is identical to that used to predict the global response of the panel in an earlier analysis by Ransom ${ }^{5}$. The finite element model used in the coupled analysis makes use of an annular mesh for the skin and rectangular meshes for the stiffeners. Interface elements are used to couple each of the stiffeners to the skin. The interface elements used in the outer two stiffeners intersect the faces of the finite elements in the skin.

Contour plots of the out-of-plane deflection, $w$, are shown in Fig. 8 superimposed on the deformed shapes of both the coupled model and the reference model. The distribution of the out-of-plane deflection at the panel mid-width is shown in Fig. 9 as a function of the longitudinal distance from one end of the panel normalized by the panel length. The distribution of the longitudinal stress resultant $N_{x}$ normalized by the applied load $\left(N_{x}\right)_{\text {avg }}$ (i.e., applied load divided by the panel width) is shown in Fig. 10 as a function of the lateral distance normalized by the cutout radius, $R$. Figures 9 and 10 indicate that the coupled analysis is in good agreement with the reference solution. The coupled analysis yields slightly different values for the longitudinal stress resultants in the region around the hole and at the skinstiffener intersection. These differences are partly due to the different discretizations used in the panel skin for the two analyses (The panel skin is slightly more refined in the coupled model than in the reference model). In any case, the results are in overall good agreement.

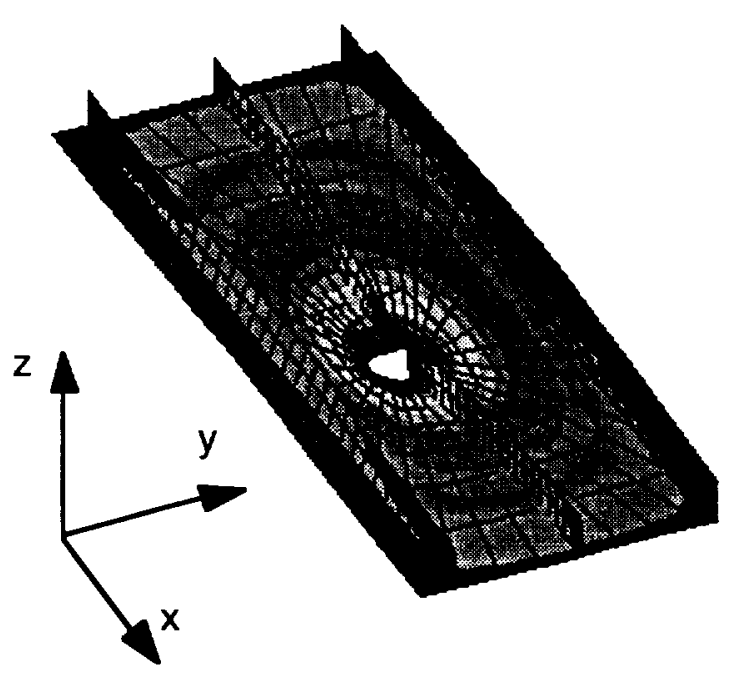

a) Coupled Model

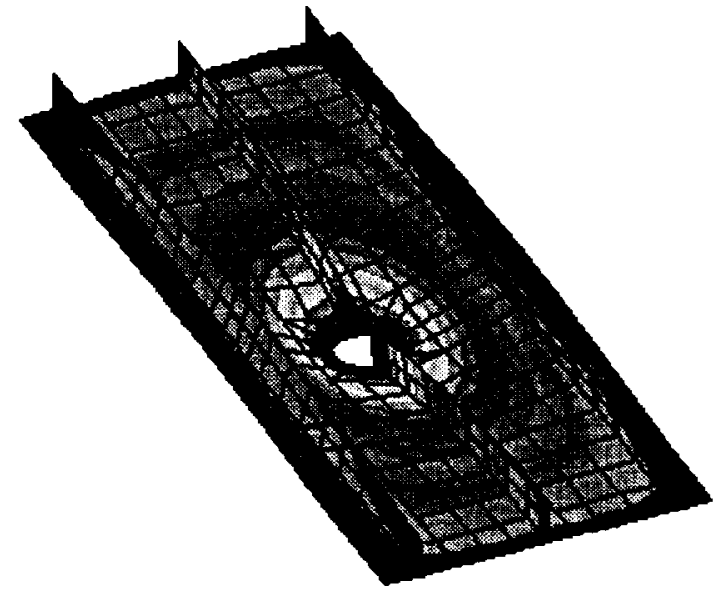

\begin{tabular}{|l} 
Max \\
Min
\end{tabular}

b) Reference Model

Figure 8. Compression-loaded composite blade-stiffened panel: the out-of plane displacement contours indicate excellent agreement between the two models 


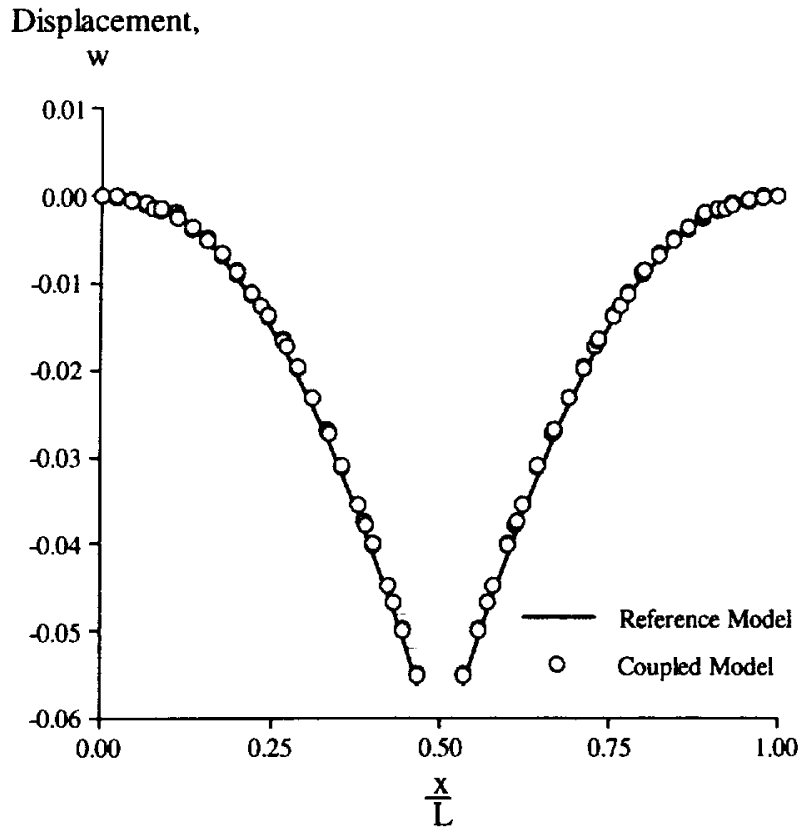

Figure 9. Normal displacement along the panel mid-width.

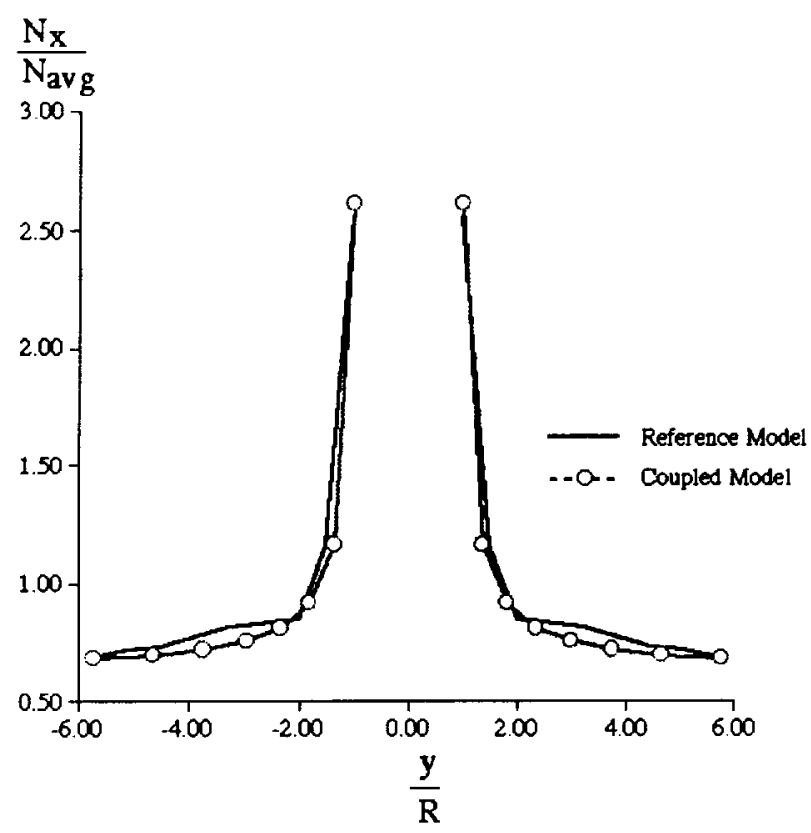

Figure 10. Axial stress distribution along the panel mid-length.

\section{Case 3: Fuselage panel with a window and two frames}

The final example shown in Fig. 11 consists of a fuselage panel with two circumferential frames. The diameter of the fuselage is 100 inches, the panel spans an arc of 34 degrees, and is 60 inches long. The thickness of the skin is 0.075 inches, and the following material properties were used: $E=10^{7}, v=0.3$. The window in the panel is 11 inches in radius and is filled with a material that is 0.3 inches thick, with a Young's modulus of $\mathrm{E}=5 \times 10^{6}$, and a Poisson's ratio of $v=0.3$. The curved edges of the panel are clamped, while the straight edges and the free length of the frames are free in the axial ( $\mathrm{z}$ ) direction and constrained in the other directions. The finite element models for the frame were generated independently of that for the skin, and the frames may be placed at any position along the axis of the fuselage. Two frame spacings are presented here. In the first model, the frames are separated by a distance of 39.6 inches. In the second model, the spacing is increased to 48.6 inches. The resulting radial displacement contours are illustrated in Figs. 12a and 12b. These models illustrate the potential use of the cross-surface interface elements in parametric studies of built-up structures.

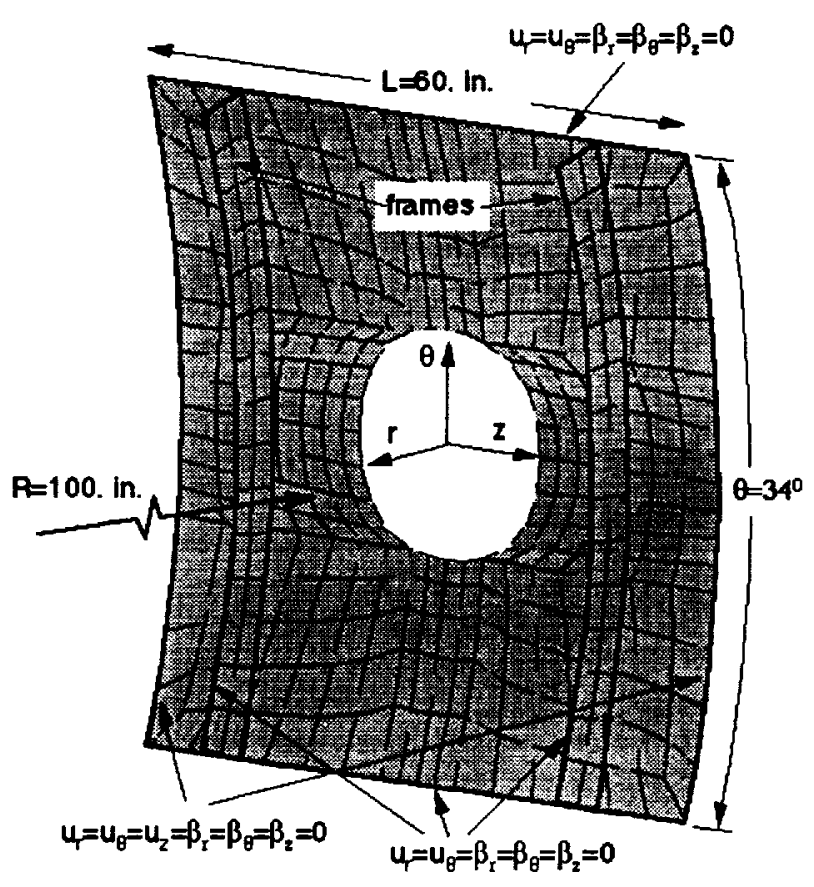

Figure 11. Model of fuselage panel with a window and two frame. 


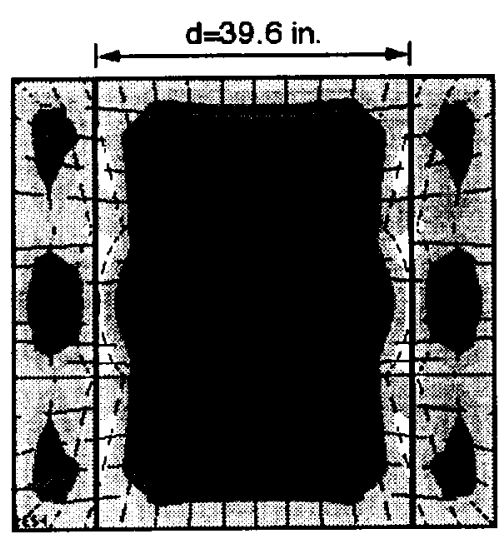

(a)

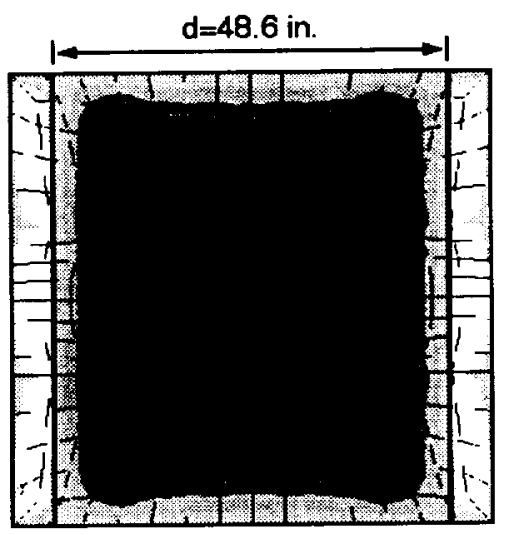

(b)

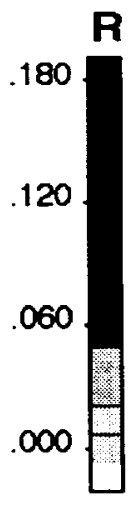

Figure 12. Radial displacement contours for two positions of the frames.

\section{Concluding Remarks}

This paper has presented the formulation of a new interface element with cross-surface capabilities. The objective of this work was to assess the potential of this technique in terms of its accuracy and, just as importantly, its ease of use. The implementation presented here is only capable of coupling across four-node shell elements. These are the simplest to develop, yet their basic stress distributions make them also the least accurate. It is expected that elements of higher order will provide better accuracy by allowing a closer approximation of the local discontinuities introduced by the interface.

The results presented herein illustrate that the interface element can produce accurate results with even relatively coarse meshes. In fact, as the mesh is refined, any desired level of accuracy can be obtained. However, the results have also shown that the convergence is not always monotonic, and that the accuracy is reduced when the load path introduces a severe discontinuity in the transverse forces on the skin elements.

A great deal of attention was given to developing this interface into a simple-to-use design tool for the quick generation of models. Therefore, even the prototype implementation of this element includes the automatic computation of the intersection path of the stiffener across the skin. The user only specifies the portion of the stiffener along which the interface is to be placed (by specifying the sequence of node numbers on the stiffener edge). A closed-form solution for the path and coupling terms is obtained for a geometric restriction: it is assumed that the interface is contained in a plane that cuts through the skin. This limitation is not overly restrictive since substructures such as bulkheads, wing ribs, or blade stiffeners, are often plane.

\section{Acknowledgments}

The authors would like to extend their gratitude to Dr. Jerrold Housner, head of the Computational Structures Branch, for his support of this work.

\section{$\underline{\text { References }}$}

1 Aminpour, M.A., Ransom, J.B., and McCleary, S.L., "Coupled Analysis of Independently Modeled Finite Element Subdomains," Paper No. 92-2235, Proceedings of the AIAA/ASME/ASCE/AHS/ASC 33rd Structures, Structural Dynamics, and Materials Conference, Dallas, TX, 1992.

2 Ransom, J.B., McCleary, S., and Aminpour, M.A., "A new interface element for connecting independently modeled substructures," Proceedings of the AIAA/ASME/ASCE/AHS 34th Structures, Structural Dynamics, and Materials Conference, La Jolla, CA, April 1993.

3 Maday, Y., Mavripilis, D., and Patera, A., "Nonconforming Mortar Element Methods: Application to Spectral Discretizations," NASA CR181729, ICASE Report No. 88-59, October 1988.

4 Shaeffer, H.G., MSC/NASTRAN Primer, Static and Normal Modes Analysis, Shaeffer Analysis, Inc., Mont Vernon, New Hampshire, 1979, pp. 262-265.

5 Ransom, J.B., Knight, N.F.,Jr., "Global/local Stress Analysis of Composite Panels," Composites and Structures, Vol 37, No. 4, pp-375-395, 1990. 


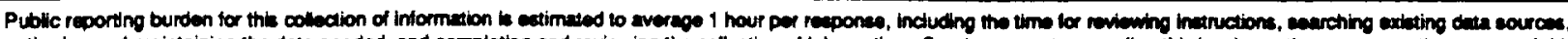

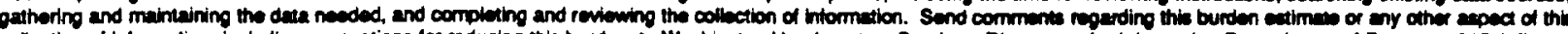

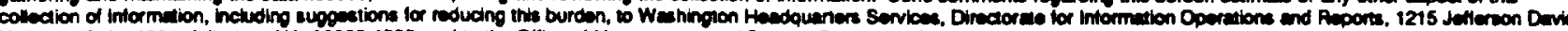

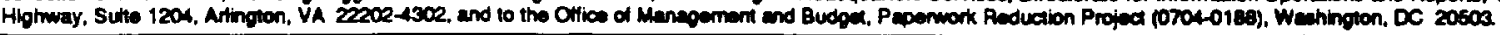
1. AGENCY USE ONLY (Laavo blank)

\begin{tabular}{|l|l|}
\hline $\begin{array}{l}\text { 2. REPORT DATE } \\
\text { July } 1994\end{array}$ & $\begin{array}{l}\text { 3. REPOAT TYPE AND DATES COVERED } \\
\text { Technical Memorandum }\end{array}$ \\
\hline
\end{tabular}

4. TITLE AND SUBTTLE

Cross-Surface Interface Element for Coupling Built-Up Structural Subdomains

WU 505-63-53-01

6. AUTHOR(S)

"C. G. Davila, J. B. Ransom, and "M. A. Aminpour

7. PERFORMING ORGANIZATKON NAME(S) AND ADDRESS(ES)

NASA Langley Research Center

PERFOAMING OAGANIZATIOI

Hampton, VA 23681-0001 REPORT NUMBER

9. SPONSORING / MONTORING AGENCY MAME(S) AND ADDRESS(ES)

National Aeronautics and Space Administration

Washington, DC 20546-0001

10. SPONSOAING / MONTOOANG AGENCY REPORT NUMBER

NASA TM-109125

\section{SUPPLEMENTARY NOTES}

"NRC Resident Research Associate; "Old Dominion University, Norfolk, VA. Paper was presented at the 35th Structures, Structural Dynamics, and Materials Conference on April 18-20, 1994, in Hilton Head, South Carolina.
12. DISTRIBUTION / AVAILABILITY STATEMENT
12b. DISTRIBUTION COOE
Unclassified - Unlimited
Subject Category 39

\section{ABSTAACT (Maximum 200 words)}

A new finite element for coupling built-up shell substructures is presented. The present work extends the hybrid variational formulation of the interface element developed by Aminpour and Ransom to permit coupling between two intersecting substructures. Designed for the assembly of independently built-up finite element models, this technique provides a level of modeling flexibility previously unavailable.

\begin{tabular}{|c|c|c|c|}
\hline \multicolumn{3}{|c|}{$\begin{array}{l}\text { 14. SUBJECT TERMS } \\
\text { Independent Modeling; Interface Element; Component Synthesis; Substructuring; } \\
\text { Built-Up Structures }\end{array}$} & \multirow{2}{*}{\begin{tabular}{|l|} 
15. NUMBER OF PAGES \\
10 \\
$\begin{array}{l}\text { 16. PRICE CODE } \\
\text { A02 }\end{array}$ \\
\end{tabular}} \\
\hline & & & \\
\hline $\begin{array}{l}\text { 17. SECURITY CLASSIFICATION } \\
\text { OF REPORT } \\
\text { Unclassified }\end{array}$ & $\begin{array}{l}\text { 18. SECUAITY CLASSIFICATION } \\
\text { OF THIS PAGE } \\
\text { Unclassified }\end{array}$ & $\begin{array}{l}\text { 19. SECURITY CLASSIFICATION } \\
\text { OF ABSTRACT } \\
\text { Unclassified }\end{array}$ & 20. UMITATION OF ABSTRACT \\
\hline
\end{tabular}

